\title{
Dynamical differential networks and modules inferring disrupted genes associated with the progression of Alzheimer's disease
}

\author{
ZHENGLING WANG $^{1^{*}}, \mathrm{XINLING} \mathrm{YAN}^{2 *}$ and CHENGHUA ZHAO ${ }^{2}$ \\ ${ }^{1}$ Office of Medical Social Work; ${ }^{2}$ Department of Neurology, \\ Yidu Central Hospital of Weifang, Weifang, Shandong 262500, P.R. China
}

Received March 31, 2016; Accepted March 6, 2017

DOI: $10.3892 /$ etm.2017.4905

\begin{abstract}
In order to understand the pathogenic factors that initiate the processes of Alzheimer's disease (AD), a method of inference of multiple differential modules $(i \mathrm{MDM})$ to conduct analysis was performed on the gene expression profile of AD. A total of 11,089 genes and 588,391 interactions were gained based on the gene expression profile and protein-protein interaction network. Subsequently, three differential co-expression networks (DCNs) were constructed with the same nodes but different interactions, and eight multiple differential modules (M-DMs) were identified. Furthermore, by performing Module Connectivity Dynamic Score to quantify the change in the connectivity of component modules, two M-DMs were identified: Module $1(\mathrm{P}=0.0419)$ and $2(\mathrm{P}=0.0419$; adjusted, $\mathrm{P} \leq 0.05)$. Finally, hub genes of $M D H 1, N D U F A B 1, N D U F B 5, D D X 1$ and MRPS35 were gained via topological analysis conducted on the $2 \mathrm{M}-\mathrm{DM}$. In conclusion, the method of $i \mathrm{MDM}$ was suitable for conducting analysis on AD. By applying $i$ MDM, 2 M-DMs were successfully identified and the $M D H 1, N D U F A B 1$, NDUFB5, DDX1 and MRPS35 genes were predicted to be important during the occurrence and development of AD.
\end{abstract}

\section{Introduction}

Alzheimer's disease (AD) is a global public health threat that continues to increase as the proportion of the population over the age of 60 rapidly increases (1). As age is the most striking risk factor for $\mathrm{AD}$, the growth of the aging population complicates this public health problem and places a strain on health care systems (2). It is known that AD is associated with perturbations of neural circuitry, which change dynamically

Correspondence to: Mrs. Zhengling Wang, Office of Medical Social Work, Yidu Central Hospital of Weifang, 4138 Linglongshannan Road, Weifang, Shandong 262500, P.R. China

E-mail: zhenglingwang36@sina.com

\section{*Contributed equally}

Key words: Alzheimer's disease, inference of multiple differential modules, multiple differential co-expression network, hub gene as the disease progresses (3). Based on the Mini-Mental State Examination, a useful index to assess cognitive function level, subjects may be categorized into four groups, as follows: Control (score $>25$ ), incipient AD (score 20-26), moderate AD (score 14-19), and severe AD (score <14) $(4,5)$. However, the pathogenic factors that initiate the processes of $\mathrm{AD}$ remain elusive.

Gene expression data have become an important resource in describing the molecular state associated with various cellular phenotypes and responses to external or internal perturbations (6). However, the probability that a false identification is made may increase when the number of tested genes increases (7). Furthermore, network-based approaches may be used to extract informative genes relying on biomolecular networks, such as the protein-protein interaction (PPI) network, gene regulatory network $(8,9)$ and gene co-expression networks (10) amongst others, rather than individual genes. In co-expression networks, two genes are connected and assumed to functionally interact if there is a correlation in their expression profiles across multiple conditions (11). However, a limitation of previous studies is that networks were only constructed using co-expression information $(6,11)$.

Inference of multiple differential modules ( $i \mathrm{MDM})$, a novel computational framework, achieves higher accuracy in inferring gene modules compared with the use of single or multiple co-expression networks (12). More specifically, $i$ MDM enables simultaneous analysis of multiple differential co-expression networks (DCNs), and is able to identify the coherently differentially-expressed genes that are either unique or shared among multiple DCNs as a module. Furthermore, a novel graph-theoretical measure is applied to quantify the dynamic changes in gene modules across conditions, and the gene modules with higher connectivity dynamics may have a greater association with the evolution and progression of the disease. This suggests that $i$ MDM may provide a method to elucidate the dynamics of gene modules and their association with the dynamics of the disease phenotype.

Therefore, in the present study, in order to further identify pathogenic factors that initiate the processes of $A D$, the $i$ MDM method was applied to conduct an analysis on AD. To achieve this, a gene expression profile of AD and human PPI data were initially obtained. Subsequently, $i$ MDM was performed to identify DCNs and candidate significant multiple differential modules (M-DMs). Finally, 
a topological analysis of M-DMs was conducted to identify hub genes, which may be important during the occurrence and development of AD.

\section{Materials and methods}

\section{Data recruiting}

Transcript data. The gene expression profile of $\mathrm{AD}$, with access number GSE1297 (5), was downloaded from the Gene Expression Omnibus database (www.ncbi.nlm.nih.gov/geo/). GSE1297 existed on the A-AFFY-33-Affymetrix GeneChip Human Genome HG-U133A [HG-U133A] platform, which was comprised of 9 normal control samples and 22 AD samples [7 incipient (score 20-26), 8 moderate (score 14-19) and 7 severe (score $<14)$ ], and the normal control samples were selected as a baseline for $\mathrm{AD}$ in the present study. The microarray data and annotation files were obtained.

In order to eliminate the influence of non-specific hybridization, a robust multichip average method (13) was used to perform background correction and a quantiles based algorithm (14) to conduct normalization. Additionally, a Micro Array Suite 5.0 algorithm (Affymetrix, Inc, Santa Clara, CA, USA) (15) was used to revise the perfect match and mismatch value, the value of which was the median value. Finally, the gene expression profile on the probe level was converted into gene symbol level using the annotate package from Bioconductor 3.4 (http://www.bioconductor.org/), duplicated symbols were removed, and a total of 12,493 gene symbols were obtained for further analysis.

PPI data. The global PPI network was integrated from the Search Tool for the Retrieval of Interacting Genes/Proteins (STRING; string-db.org/). In the STRING, there were a total of 787,896 interactions (16,730 genes) of human beings. Based on the preprocessed gene expression profile of $\mathrm{AD}$, a total of 11,089 genes were mapped onto the global PPI network. In addition, the corresponding gene associations were selected from the PPI network, and a total of 588,391 interactions were obtained.

\section{iMDM method}

DCN construction. For each of the three stages of AD (incipient, moderate and severe), two steps were performed to construct the DCN. Initially, a binary co-expression network was built. To gain the co-expressed genes, Pearson correlation coefficients (PCC) (16) were implemented to calculate the correlation of each gene pair in the disease. In the present study, for each stage, edges of with absolute values of PCC $\geq 0.9$ were separately selected to construct the binary co-expression network.

The second step was that each edge of the binary co-expression network was assigned a weight value based on the differential gene expression between the disease of each stage and the baseline. In the present study, EdgeR V3.16.5 in Bioconductor (17), which is used for differential expression analysis of digital gene expression data, was utilized to detect differential gene expression for the microarray data. Prior to analysis with EdgeR, the P-values of differential gene expression among stages and the baseline condition were obtained using a one-sided Student's t-test. Subsequently, the weight $\left(w_{\mathrm{x}, \mathrm{y}}\right)$ on edge $(\mathrm{x}, \mathrm{y})$ in the differential network was calculated as follows:

$$
w_{x, y}=\left\{\begin{array}{cl}
\frac{\left(\log p_{x}+\log p_{y}\right)^{1 / 2}}{\left(2 * \max _{k \in N}\left|\log p_{k}\right|\right)^{1 / 2}}, & \text { if } \operatorname{cor}(x, y) \geq \delta, \\
0, & \text { if } \operatorname{cor}(x, y)<\delta,
\end{array}\right.
$$

Where $p_{\mathrm{x}}$ and $p_{\mathrm{y}}$ were $\mathrm{P}$-values of differential expression for the $\mathrm{x}$ and $\mathrm{y}$ genes $(\mathrm{P}<0.05$ was considered to indicate a statistically significant difference), respectively, $\mathrm{N}$ was the node set of the co-expression network, and $\operatorname{cor}(x, y)$ was the absolute value of PCC between the $\mathrm{x}$, y genes based on their expression profiles.

Under this weighting scheme, genes that were co-expressed and significantly differentially expressed were assigned higher weights, which satisfied the assumption of the present study that those genes likely to participate in a pathway that exhibited differential activities between the two conditions being compared. $\mathrm{P}<0.05$ was considered to indicate a statistically significant difference. Therefore, three DCNs for the three stages of $\mathrm{AD}$ were separately built based on the analysis above.

Identification of M-DMs in multiple DCNs. After each edge was given a weight value, candidate M-DMs were mined. The M-module algorithm, which was designed for identifying gene modules with common members but varied connectivity across multiple molecular interaction networks, was applied to identify M-DMs (18). Furthermore, the M-DM search consisted of three steps: Seed prioritization, module search by seed expansion and refinement of candidate modules.

Seed prioritization to seek seed genes. Initially, the genes contained in the DCNs were ranked based on the degree centrality features of the genes in the network. More specifically, for each network $G_{m}=\left(N, E_{m}\right)(1 \leq m \leq M)$ with an adjacency matrix $\mathrm{A}_{\mathrm{i}}$, the importance of the gene $x$ in each DCN was calculated according to the following formula:

$$
g(x)=\sum_{y \in N_{i}(x)} A_{x y i}^{\prime} g(y)
$$

Where $g(x)$ denoted the importance of vertex $x$ in the corresponding network, $N_{\mathrm{i}}(x)$ denoted the set of neighbors of $x$ in $\mathrm{G}_{\mathrm{m}}$ and $A_{\mathrm{i}}^{\prime}$ denoted the degree normalized weighted adjacency matrix. The product A'-g denoted the information propagation on network via the edges of networks, which meant the importance of a node was associated with the number of its neighbors, strength of connection and importance of its neighbors.

According to the computational formula given above, the importance of the genes in all individual networks was obtained, which were denoted as z-score values. Subsequently, all z-score values were ranked in descending order in all individual networks, and the rank for that gene was obtained across all networks by calculating the mean z-scores across all networks. The top 5\% of genes, with the highest z-scores, were selected as seed genes.

M-DMs searching. Initially, each seed gene was selected as a differential module, $S$. Next, starting from each seed gene, $x$, the network gene $y$ that was adjacent to $x$ was added to $S$ 
to form a module $S^{\prime}$. The entropy decrease between these two modules was calculated according to the following formula:

$$
\Delta H\left(S^{\prime}, S\right)=H(S)-H\left(S^{\prime}\right)
$$

$\Delta \mathrm{H}\left(\mathrm{S}^{\prime}, \mathrm{S}\right)>0$ indicated that the addition of vertex $y$ improved the connectivity of the former M-module, S. Furthermore, genes were iteratively added to module $S$ that were adjacent to $x$ until there was no decrease in the objective function of $\Delta \mathrm{H}$. In this case, all genes were connected together to form M-DMs.

Refinement of candidate modules. In the refinement step, only those M-DMs with sizes $\geq 5$ were kept. In addition, the ratio of intersection over union for two sets was measured by the Jaccard index, and the two sets were merged into a module with a Jaccard index $\geq 0.5$.

Calculation of the statistical significance of candidate $M-D M s$. To further analyze the statistical significance of candidate M-DMs, randomized networks were constructed based on the null score distribution of M-DMs. A module search was performed on the randomized networks according to the methods mentioned above. Each network was completely randomized 100 times. Furthermore, the empirical P-value of an M-DM was calculated as the probability of the module having the observed score or smaller by chance. Furthermore, the Benjamini and Hochberg (19) method was performed to conduct multiple testing on the P-values. The modules with $\mathrm{P} \leq 0.05$ were considered as M-DMs.

Quantification of connectivity dynamics of shared M-DMs. A graph-theoretical measure, the Module Connectivity Dynamic Score (MCDS) was utilized for the M-DMs that had multiple component modules from different DCNs in order to quantify the change in the connectivity of the component modules. Briefly, given a dynamic module (C) whose weighted adjacent matrices of the corresponding induced subgraphs were denoted by $A_{x}^{c}(1 \leq \mathrm{x} \leq \mathrm{M})$, the MCDS between two adjacent component modules was defined as the $\mathrm{L}_{2}$ norm of the matrix subtraction normalized by the number of genes in the M-DM, for example,

$$
\Delta A_{x, x+1}^{C}=\left\|A_{x}^{C}-A_{x+1}^{C}\right\|_{2} /|C|
$$

where \|\|$_{2}$ was the matrix $L_{2}$ norm. Furthermore, the overall MCDS of an M-DM was defined as the mean MCDS of all pairwise comparisons:

$$
\sigma\left(A^{C}\right)=\sum_{x=1}^{M-1} \Delta A_{x, x+1}^{C} /(M-1)
$$

The statistical significance of MCDS for an M-DM was computed in a similar way as that for M-DMs, and Benjamini-Hochberg was used to conduct multiple testing corrections. The modules were adjusted and values with $\mathrm{P} \leq 0.05$ were considered as differential modules with significant connectivity dynamics.

Topological analysis of M-DMs. Each M-DM with higher quantification of connectivity dynamics was considered as a sub-network, and topological analysis of differential modules was conducted in order to further investigate the biological functions and significance of nodes in the differential modules, indices of topological analysis [degree (20), closeness (21), betweenness (22) and shortest path length (23)], and were performed to characterize the importance of the nodes. Traditionally, nodes with a high degree (highly connected) are called 'hubs', which interact with several other genes, suggesting a central role in the interaction network. In the present study, the top $10 \%$ of the genes according to the degree centralities were considered as hub genes.

\section{Results}

DCN constructions. In the present study, an analysis was conducted on the gene expression profile of AD to further elucidate its molecular mechanism. After having selected the gene expression profile that contained the interactions of the PPI network, the absolute value of the PCC of the expression profiles of each gene interaction in each stage was calculated. In total, three DCNs, one for each stage of AD, were built separately. It was revealed that the nodes in these DCNs were the same, whereas the interactions were different from each other in the three stages of AD. Immediately after, the EdgeR package was utilized to assign weight values based on differential gene expression between the different stages of AD and the baseline, respectively. In this case, three DCNs that had the same nodes but different interactions were identified.

Identification of M-DMs in multiple DCNs. Initially, the genes contained in the DCNs were ranked according to the degree centrality values in each DCN. Degree centrality values were subsequently converted into z-score values, and ranked for that gene across all networks by calculating the mean z-score across all networks. Furthermore, the top 5\% genes with the highest $\mathrm{z}$-scores were selected as seed genes, and 28 seed genes were obtained (Table I).

Beginning with each seed gene, modules were searched for each stage. By removing those M-DMs with sizes $<5$, as well as merging the two sets with Jaccard index $\geq 0.5,17$ modules were identified. These modules were regarded as candidate M-DMs. To further analyze the statistical significance of candidate M-DMs, a randomized test method was applied to conduct the analysis, and the Benjamini-Hochberg method was performed to conduct multiple testing on the P-values. By setting the threshold value of adjusted $\mathrm{P} \leq 0.05$, eight $\mathrm{M}-\mathrm{DMs}$ were gained.

Quantification of connectivity dynamics of shared M-DMs. For the eight M-DMs that had multiple component modules from different DCNs, MCDS was performed to quantify the change in the connectivity of the component modules. Under adjusted $\mathrm{P} \leq 0.05$, two M-DMs: Module $1(\mathrm{P}=0.0419)$ and 2 $(\mathrm{P}=0.0419)$ exhibited significant connectivity dynamics across multiple conditions $(\mathrm{P}<0.05)$. These two M-DMs were regarded to be closely associated with AD. As shown in Figs. 1 and 2, there were 88 nodes ( 884 edges) in module 1 and 89 nodes (731 edges) in module 2. As observed from Figs. 1 and 2, the gene composition of each module in the 
Table I. Z-score values of the 28 seed genes.

\begin{tabular}{|c|c|c|c|c|}
\hline Gene symbol & Z-score 1 (incipient AD) & Z-score 2 (moderate AD) & Z-score 3 (severe AD) & Average \\
\hline NDUFB5 & $1,003.556$ & $1,437.713$ & $2,098.125$ & $1,513.132$ \\
\hline DDX1 & 905.415 & $1,359.723$ & $1,809.015$ & $1,358.051$ \\
\hline NDUFAB1 & 854.492 & $1,262.754$ & $1,610.130$ & $1,242.459$ \\
\hline ACTR6 & 802.895 & $1,034.827$ & $1,646.069$ & $1,161.264$ \\
\hline C14orf166 & 794.725 & $1,102.397$ & $1,426.624$ & $1,107.916$ \\
\hline ACTR10 & 694.259 & $1,063.502$ & $1,541.512$ & $1,099.757$ \\
\hline MRPS35 & 694.404 & $1,000.464$ & $1,508.590$ & $1,067.819$ \\
\hline HSPE1 & 650.006 & $1,122.219$ & $1,431.178$ & $1,067.801$ \\
\hline MDH1 & 602.146 & $1,125.608$ & $1,417.861$ & $1,048.538$ \\
\hline NDUFB3 & 713.060 & $1,035.019$ & $1,391.509$ & $1,046.529$ \\
\hline SLIRP & 688.469 & $1,007.552$ & $1,440.883$ & $1,045.634$ \\
\hline DLD & 617.417 & 913.604 & $1,460.811$ & 997.277 \\
\hline COPS5 & 661.617 & 978.652 & $1,332.187$ & 990.819 \\
\hline PSMD10 & 635.760 & 927.430 & $1,383.522$ & 982.237 \\
\hline LAMTOR3 & 753.325 & 913.979 & $1,254.756$ & 974.020 \\
\hline ATP5F1 & 618.705 & 942.688 & $1,325.607$ & 962.333 \\
\hline SNRNP27 & 628.393 & 882.229 & $1,349.686$ & 953.436 \\
\hline EIF3J & 730.916 & 800.174 & $1,284.984$ & 938.691 \\
\hline COX7C & 596.897 & 883.640 & $1,234.575$ & 905.037 \\
\hline PTGES3 & 742.810 & 752.692 & $1,160.379$ & 885.293 \\
\hline DYNLL1 & 531.537 & 934.580 & $1,125.114$ & 863.744 \\
\hline PCMT1 & 570.177 & 785.947 & $1,145.326$ & 833.816 \\
\hline PSMB1 & 587.862 & 799.568 & $1,106.171$ & 831.200 \\
\hline SRP9 & 581.777 & 759.436 & $1,136.389$ & 825.867 \\
\hline DPM1 & 572.269 & 725.154 & $1,092.859$ & 796.761 \\
\hline METTL5 & 516.985 & 830.050 & 999.440 & 782.158 \\
\hline POMP & 526.684 & 757.540 & 996.599 & 760.275 \\
\hline GHITM & 474.148 & 787.383 & 988.797 & 750.110 \\
\hline
\end{tabular}

Z-score 1 corresponds to incipient AD; Z-score 2 corresponds to moderate AD; Z-score 3 corresponds to severe AD.
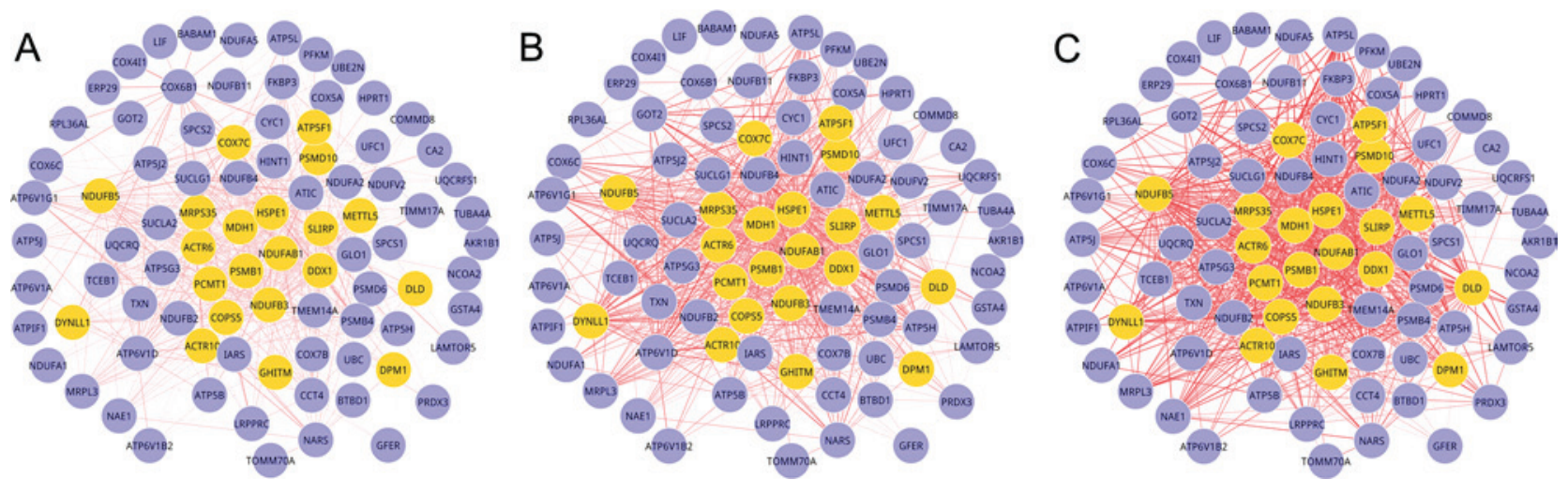

Figure 1. Multiple differential modules of module 1 for the three stages of AD. (A) Incipient, (B) moderate and (C) severe AD. Yellow nodes represent the seed genes, and the thickness of the edges represented the strength of the interactions between the nodes. AD, Alzheimer's disease.

three stages was the same, but the edges varied. As the edge weight in DCNs was a measure of differential gene expression between the disease and normal control conditions, a larger mean edge weight of an M-DM indicated a greater difference in the expression of module genes. Therefore, after investigating the root tracing, the dynamics of the M-DMs resulted from the gene expression value of the genes in different stages of the AD. 
Table II. Topological properties of the hub genes in module 1.

\begin{tabular}{|c|c|c|c|c|}
\hline Gene & Degree & Closeness & Betweenness & Shortest Path Length \\
\hline MDH1 & 78 & 0.906250 & 0.25422146 & 1.10344828 \\
\hline NDUFAB1 & 65 & 0.776786 & 0.06621343 & 1.28735632 \\
\hline NDUFB5 & 64 & 0.769912 & 0.05491728 & 1.29885057 \\
\hline SLIRP & 52 & 0.696000 & 0.02372897 & 1.43678161 \\
\hline DDX1 & 51 & 0.690476 & 0.02436002 & 1.44827586 \\
\hline HSPE1 & 50 & 0.685039 & 0.02535913 & 1.45977011 \\
\hline NDUFB3 & 47 & 0.669231 & 0.01829438 & 1.49425287 \\
\hline MRPS35 & 44 & 0.654135 & 0.01638579 & 1.52873563 \\
\hline
\end{tabular}

Table III. Topological properties of the hub genes in module 2.

\begin{tabular}{|c|c|c|c|c|}
\hline Gene & Degree & Closeness & Betweenness & Shortest Path Length \\
\hline DLD & 80 & 0.916667 & 0.326576 & 1.090909 \\
\hline NDUFB5 & 55 & 0.709677 & 0.060582 & 1.409091 \\
\hline ACTR6 & 50 & 0.682171 & 0.078818 & 1.465909 \\
\hline NDUFAB1 & 46 & 0.661654 & 0.028408 & 1.511364 \\
\hline MRPS35 & 45 & 0.656716 & 0.029722 & 1.522727 \\
\hline DDX1 & 43 & 0.661654 & 0.034592 & 1.511364 \\
\hline PTGES3 & 39 & 0.624113 & 0.02588 & 1.602273 \\
\hline MDH1 & 37 & 0.619718 & 0.02162 & 1.613636 \\
\hline
\end{tabular}
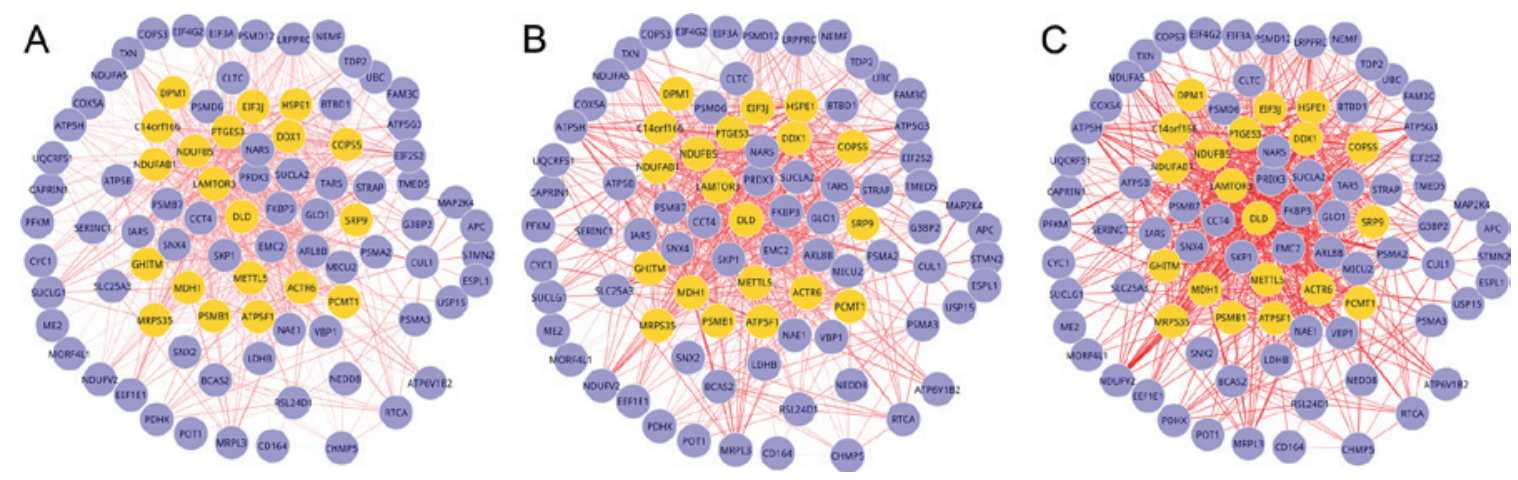

Figure 2. Multiple differential modules of module 2 for the three stages of AD. (A) Incipient, (B) moderate and (C) severe AD. Yellow nodes represent the seed genes, and the thickness of the edges represented the strength of the interactions between the nodes. AD, Alzheimer's disease.

Topological analysis of $M-D M s$. Since it was indicated that the dynamics of the M-DMs resulted from the genes, M-DMs with higher quantification of connectivity dynamics were separately considered as a network in order to further disclose the molecular mechanism of the AD. Topological analysis was conducted on them to further to investigate the biological functions and significance of the nodes in the differential modules. In these DMs, the nodes and the number of edges were the same in all stages of AD, and only the edge values were different. Therefore, the degree centralities were the same in different stages of AD. As the genes were ranked in descending order according to the degree centralities, the top $10 \%$ of the genes were considered as hub genes. For module 1,
8 hub genes were obtained as shown in Table II: $M D H 1$ (degree=78), NDUFAB1 (degree=65), NDUFB5 (degree=64), SLIRP (degree=52), DDX1 (degree=51), HSPE1 (degree=50), NDUFB3 (degree=47) and MRPS35 (degree=44). A total of 8 hub genes were also obtained for module 2 as shown in Table III: DLD (degree=80), NDUFB5 (degree=55), ACTR6 (degree=50), NDUFAB1 (degree=46), MRPS35 $($ degree=45), DDX1 (degree=43), PTGES3 (degree=39) and $M D H 1$ (degree=37). Additionally, it was identified that all hub genes were also seed genes. Furthermore, MDH1, NDUFAB1, NDUFB5, DDX 1 and MRPS35 were hub genes in both modules 1 and 2, and it was predicted that they may have close association during the occurrence and development of AD. 


\section{Discussion}

Network biology has been proven to be a powerful tool for analyzing and representing complex molecular networks. From a systems biology point of view, diseases are caused by perturbations to the gene network, which change dynamically during the progression of the disease (24). As genes present in the same pathway tend to have correlated expression, analyzing a co-expression network is an effective strategy for pathway inference (25). Furthermore, complex diseases such as $\mathrm{AD}$ are associated with a continuum of molecular events that begin with the early initiation events through progression and catastrophic end-stage events. Although analyzing and understanding disease-stage-specific molecular events are critical for understanding disease etiology and development of therapeutic interventions, the current knowledge on the dynamics of gene networks during disease progression is rather limited. Many previous studies on connectivity dynamics have been focused on the dynamics of hub genes, such as using integrative network analysis to both identify candidate driver genes and candidate pathways (26). Vandin et al (27) have used a network diffusion algorithm to identify sub-networks enriched for mutations within a large gene interaction network. Additionally, studies have also investigated the network properties of complex disease alterations, and have noted that complex disease alterations tend to cluster within closely knit network modules or communities, and that altered modules are associated with specific biological pathways $(28,29)$. However, there were two problems for understanding network dynamics; identifying the sub-networks that exhibit structural changes in response to different growth or developmental events, or different spatial location and quantifying the dynamic changes in the sub-network structure (18). Using $i \mathrm{MDM}$, only sets of genes that are differentially expressed as well as those that exhibit correlated expression patterns under diseased states will be identified as a module, so that the entire pathway consists of perturbed genes under disease condition.

In the present study, $i$ MDM was applied as a novel method to conduct analysis on the gene expression profile of $\mathrm{AD}$, and two M-DMs were identified: Module $1(\mathrm{P}=0.0419)$ and $2(\mathrm{P}=0.0419)$, which had higher quantification of connectivity dynamics. In order to understand the association between the genes and $\mathrm{AD}$ and because the dynamics of the M-DMs resulted from the gene expression value of the genes at different stages of the $\mathrm{AD}$, a topological analysis was conducted on these two M-DMs. It was revealed that $M D H 1$, NDUFAB1, NDUFB 5, DDX1 and MRPS35 were hub genes in both modules 1 and 2. Furthermore, it was predicted that these genes may have a close association during the occurrence and development of AD. In order to further expose the correlation between these genes and $\mathrm{AD}$, the $\mathrm{MDHl}$ gene was selected as an example.

$M D H 1$, which encodes soluble cytosolic malate dehydrogenase (MDH1), is important in transporting nicotinamide adenine dinucleotide equivalents across the mitochondrial membrane, controlling the tricarboxylic acid cycle pool size and providing contractile function (30). The enzyme that functionally connects glutamate dehydrogenase (GDH) and MDH1 is aspartate aminotransferase (AAT). GDH is co-localized in cells with AAT, which participates in the malate-aspartate shuttle with MDHs (31). Previous studies have indicated that, under AD conditions, the activity of $\mathrm{MDH}$ is increased in the brain (32), and the activity also increases with age in a rat brain (33). Furthermore, Korolainen et al (34) have indicated that the total amount of soluble MDH1 is unchanged in $\mathrm{AD}$ when compared with controls, indicating differences in the amounts of distinctive isoforms. The most likely explanations are aberrant post-translational modification of MDH1 in AD leading to a differential quantitative distribution of isoforms when compared with controls. However, the most notable result, from the study of Korolainen et al (34), was that GDH and MDH1, which were closely associated with neurotransmitter release and energy metabolism, were less oxidized in $\mathrm{AD}$, compared with controls. In the present study, $M D H 1$ was identified as a hub gene in both M-DMs, which suggests that it serves an important role in AD.

In conclusion, in the present study a method of $i \mathrm{MDM}$ was performed in order to conduct analysis on $\mathrm{AD}$. By applying $i \mathrm{MDM}$, two M-DMs were successfully identified, and the MDH1, NDUFAB1, NDUFB5, DDX1 and MRPS35 genes were predicted to have key roles during the occurrence and development of AD. Further experimental verification will be conducted to verify the results of the present study.

\section{Acknowledgements}

The present study was supported by the Office of Medical Social Work and Department of Neurology, Yidu Central Hospital of Weifang (Weifang, China). The authors would like to thank all members of the research group. Furthermore, the authors are grateful to Beijing Springer Medical Research Institute for providing professional translation and manuscript editing services.

\section{References}

1. Glenner GG and Wong CW: Alzheimer's disease: Initial report of the purification and characterization of a novel cerebrovascular amyloid protein. 1984. Biochem Biophys Res Commun 425: 534-539, 2012.

2. Alzheimer's Association: 2014 Alzheimer's disease facts and figures. Alzheimers Dement 10: e47-e92, 2014.

3. Leal SL and Yassa MA: Perturbations of neural circuitry in aging, mild cognitive impairment, and Alzheimer's disease. Ageing Res Rev 12: 823-831, 2013.

4. Clark CM, Sheppard L, Fillenbaum GG, Galasko D, Morris JC, Koss E, Mohs R and Heyman A: Variability in annual mini-mental state examination score in patients with probable Alzheimer disease: A clinical perspective of data from the consortium to establish a registry for Alzheimer's disease. Arch Neurol 56: 857-862, 1999.

5. Blalock EM, Geddes JW, Chen KC, Porter NM, Markesbery WR and Landfield PW: Incipient Alzheimer's disease: Microarray correlation analyses reveal major transcriptional and tumor suppressor responses. Proc Natl Acad Sci USA 101: 2173-2178, 2004.

6. Carter SL, Brechbühler CM, Griffin M and Bond AT: Gene co-expression network topology provides a framework for molecular characterization of cellular state. Bioinformatics 20: 2242-2250, 2004.

7. Dudoit S, Yang YH, Callow MJ and Speed TP: Statistical methods for identifying differentially expressed genes in replicated cDNA microarray experiments. Stat Sin 12: 111-139, 2002.

8. Jeong H, Mason SP, Barabási AL and Oltvai ZN: Lethality and centrality in protein networks. Nature 411: 41-42, 2001.

9. Teichmann SA and Babu MM: Gene regulatory network growth by duplication. Nat Genet 36: 492-496, 2004. 
10. Stuart JM, Segal E, Koller D and Kim SK: A gene-coexpression network for global discovery of conserved genetic modules. Science 302: 249-255, 2003.

11. Zhang B and Horvath S: A general framework for weighted gene co-expression network analysis. Stat Appl Genet Mol Biol 4: Article17, 2005.

12. MaX,GaoL, Karamanlidis G, Gao P,LeeCF, Garcia-MenendezL, Tian $\mathrm{R}$ and Tan K: Revealing pathway dynamics in heart diseases by analyzing multiple differential networks. PLoS Comput Biol 11: e1004332, 2015.

13. Ma L, Robinson LN and Towle HC: ChREBP*Mlx is the principal mediator of glucose-induced gene expression in the liver. J Biol Chem 281: 28721-28730, 2006.

14. Rifai N and Ridker PM: Proposed cardiovascular risk assessment algorithm using high-sensitivity $\mathrm{C}$-reactive protein and lipid screening. Clin Chem 47: 28-30, 2001.

15. Pepper SD, Saunders EK, Edwards LE, Wilson CL and Miller CJ: The utility of MAS5 expression summary and detection call algorithms. BMC Bioinformatics 8: 273, 2007.

16. Benesty J, Chen J, Huang Y and Cohen I: Pearson correlation coefficient. Noise reduction in speech processing: 1-4, 2009.

17. Robinson MD, McCarthy DJ and Smyth GK: edgeR: A bioconductor package for differential expression analysis of digital gene expression data. Bioinformatics 26: 139-140, 2010

18. Ma X, Gao L and Tan K: Modeling disease progression using dynamics of pathway connectivity. Bioinformatics 30 : 2343-2350, 2014

19. Benjamini Y and Hochberg Y: Controlling the false discovery rate: A practical and powerful approach to multiple testing. J R Stat Soc 57: 289-300, 1995.

20. Haythornthwaite C: Social network analysis: An approach and technique for the study of information exchange. Libr Inf Sci Res 18: 323-342, 1996.

21. Wasserman S and Faust K: Social network analysis: Methods and applications. Struct Anal Soc Sci 8, 1994.

22. Barthelemy $M$ : Betweenness centrality in large complex networks. Eur Phys J B 38: 163-168, 2004.

23. Chuang TN and Kung JY: The fuzzy shortest path length and the corresponding shortest path in a network. Comput Oper Res 32: $1409-1428,2005$.
24. Ellis JD, Barrios-Rodiles M, Colak R, Irimia M, Kim T, Calarco JA, Wang X, Pan Q, O'Hanlon D, Kim PM, et al: Tissue-specific alternative splicing remodels protein-protein interaction networks. Mol Cell 46: 884-892, 2012.

25. D'haeseleer P, Liang S and Somogyi R: Genetic network inference: From co-expression clustering to reverse engineering. Bioinformatics 16: 707-726, 2000.

26. Torkamani A and Schork NJ: Identification of rare cancer driver mutations by network reconstruction. Genome Res 19: 1570-1578, 2009.

27. Vandin F, Upfal E and Raphael BJ: Algorithms for detecting significantly mutated pathways in cancer. J Comput Biol 18 506-521, 2010.

28. Cerami E, Demir E, Schultz N, Taylor BS and Sander C: Automated network analysis identifies core pathways in glioblastoma. PLoS One 5: e8918, 2010.

29. Wu G, Feng X and Stein L: Research a human functional protein interaction network and its application to cancer data analysis. Genome Biol 11: R53, 2010

30. Lo AS, Liew CT, Ngai SM, Tsui SK, Fung KP, Lee CY and Waye MM: Developmental regulation and cellular distribution of human cytosolic malate dehydrogenase (MDH1). J Cell Biochem 94: 763-773, 2005.

31. Kimmich GA, Roussie JA and Randles J: Aspartate aminotransferase isotope exchange reactions: Implications for glutamate/glutamine shuttle hypothesis. Am J Physiol Cell Physiol 282: C1404-C1413, 2002.

32. Op den Velde W and Stam FC: Some cerebral proteins and enzyme systems in Alzheimer's presenile and senile dementia. J Am Geriatr Soc 24: 12-16, 1976.

33. Malik P, McKenna MC and Tildon JT: Regulation of malate dehydrogenases from neonatal, adolescent and mature rat brain. Neurochem Res 18: 247-257, 1993.

34. Korolainen MA, Goldsteins G, Nyman TA, Alafuzoff I, Koistinaho $\mathrm{J}$ and Pirttilä T: Oxidative modification of proteins in the frontal cortex of Alzheimer's disease brain. Neurobiol Aging 27: 42-53, 2006. 